\title{
Gender, motherhood, and the perception of just earnings: Results of a multifactorial (vignette) study
}

\author{
Kinga Wysieńska-Di Carlo and Zbigniew Karpiński \\ Institute of Philosophy and Sociology \\ Polish Academy of Sciences*
}

January 2018

\begin{abstract}
Gender pay gaps and wage penalties for mothers are well-established phenomena. Their persistence may be partially explained by the fact that the unequal distribution of rewards between genders is often perceived as just and legitimate. The goal of our study was to establish to what extent and under what conditions motherhood and fatherhood status affect perceptions of just inequalities. Using expectation states theories, we predicted that there would be greater acceptance of the unequal distribution of rewards between women and men with the same job experience in top-level occupations compared with bottom and mid level occupations. We also predicted that being a male or female parent would further accentuate the relationship between gender and occupational prestige, leading to acceptance of even greater income gaps between mothers and fathers. A vignette study conducted in 2017 on a representative sample of Polish respondents active in the labor force, which yielded approximately 38,000 observations, provides evidence partially conforming to our predictions. That is, willingness to tolerate differences in just earnings for men and women is higher in the high occupational status categories than in the medium and low occupational status categories. The effects of parenthood are less clear. Although the legitimized difference in earnings varies by age and parenthood status, we found rather moderate levels of motherhood penalties and much higher premiums for fatherhood, especially at the early stages of men's careers. In addition, contrary to our predictions, mothers in high-prestige occupations were not penalized more than mothers in low and medium prestige occupations. Perceptions of legitimized inequalities between genders were independent of respondents' characteristics.
\end{abstract}

\section{Funding}

The work was supported by the Polish National Science Center (grant numbers 2015/19/B/HS6/03169 and $2017 / 25 / \mathrm{B} / \mathrm{HS} 6 / 02697)$.

Draft prepared for submission to the 2018 Annual Meeting of the American Sociological Association.

\section{Introduction}

Evidence that having children has a negative effect on women's wages and career prospects is ubiquitous (e.g., Abendroth, Huffman, and Treas 2014; Budig and England 2001; Budig, Misra, and Boeckmann 2016; Gangl and Ziefle 2009; Susan and Jane 2003). Men, on the other hand, experience fatherhood premiums (e.g., Glauber 2008; Hodges and Budig 2010; Koslowski 2010; Petersen, Penner, and Høgsnes 2014). The size of motherhood penalty and fatherhood premium varies across countries (e.g., Aisenbrey, Evertsson,

*Corresponding author: kwysienska@ifispan.waw.pl. This manuscript is a work in progress. Please do not quote without permission. 
and Grunow 2009; Cooke 2014), welfare regimes (e.g., Budig, Misra, and Boeckmann 2016; Evertsson and Grunow 2012), and the distribution of income and occupational prestige (Budig and Hodges 2010; England et al. 2016). It has been demonstrated that women at the upper end of the income distribution and in high prestige and highly demanding occupations incur larger income losses per child than do women in medium and low prestige occupations and women with lower incomes (England et al. 2016; Magnusson 2010).

Researchers offer various explanations regarding the mechanisms producing parenthood related penalties and premiums. Depending on whether the focus is on explaining wage differentials between men and women within or between households, as well as on whether there are controls for family status and partner's income, possible explanations include: mechanisms of selection into parenthood processes; household specialization/division of labor; or mechanisms related to human capital accumulation/loss and employer's discrimination. The last explanation particularly has garnered researchers' attention in recent years (Bygren, Erlandsson, and Gähler 2017; Correll, Benard, and Paik 2007; Ridgeway and Correll 2004; Ridgeway 2011, pp. 117-119). It proposes that motherhood constitutes a status characteristic. That is, being a female parent is linked to a set of universally shared beliefs regarding competences, motivations, and general worth. These beliefs are hypothesized to exert an especially strong impact in employment settings, as being a mother is perceived to be in direct conflict with being an "ideal worker." Mothers are believed to be less committed to their workplaces, less competent, and generally less productive (e.g., Correll, Benard, and Paik 2007; Ridgeway and Correll 2004).

Motherhood and gender as status characteristics may also jointly affect perceptions of just earnings, which could partially explain the existence and persistence of motherhood pay penalties. Jasso and Webster (1997) and Auspurg, Hinz, and Sauer (2017) have demonstrated that perceived fair rewards for men and women indeed differ and favor men. However, the impact of motherhood on perceptions of just rewards has not, to our knowledge, been established. The purpose of our study is to fill that gap, and explain and empirically assess how gender and motherhood affect evaluations of just pay, as well as determine how their impact varies under different conditions. Applying status characteristics, double standards, and reward expectations theory, we describe and predict the conditions under which the unequal distribution of rewards

between members of socially valued categories, such as genders, will be perceived as just and legitimate. The conditions that we predict will be most relevant are status consistency or inconsistency between genders on the one hand, and, on the other hand, occupational status and years of experience. We also predict that an additional factor, parenthood, will accentuate the relationship between gender and the aforementioned characteristics. More specifically, we propose that there will be greater acceptance of unequal distribution of rewards between women and men in top-level occupations (status inconsistency), and less acceptance of inequalities in bottom and medium level occupations (status consistency). This relationship will be mediated by parenthood status of the hypothetical male and female earners. That is, there will be stronger acceptance of unequal pay between mothers and fathers in high status occupations than between men and women without children in the same types of jobs. In lower status occupations, on the other hand, gaps between mothers and fathers will still be more acceptable than those between men and women without children, but the discrepancy will be far smaller.

In the next sections, we provide the theoretical rationale for our reasoning, describe our methods and analytical approach, and discuss our results. We conclude with a brief description of a follow-up study that is in progress.

\section{Motherhood and fatherhood as status characteristics - empirical evidence}

A growing body of research indicates (directly or indirectly) that motherhood carries status value. For example, Ridgeway and Correll (2004) provide a review of evidence indicating that, when information about motherhood is available in a work context, it negatively affects evaluations of mothers' competence and suitability for positions of authority. They also argue that motherhood will have a stronger effect than gender in this context, due to strong cultural beliefs that taking care of a child is in direct conflict with being a good worker. Correll, Benard, and Paik (2007) tested this conjecture using both laboratory and natural experiments, and found that mothers indeed were evaluated as less competent and committed, and, as a consequence, were offered lower salaries, and experienced more hiring discrimination by actual employers than 
did men and women without children. Fathers, on the other hand, were evaluated as more committed and offered higher salaries, and were preferred by actual employers over both childless men as well as mothers (but not childless women). Baert (2014) provides further (although indirect) evidence for hiring discrimination against (potential) mothers. Results of his field experiment, which employed the paired-resume design to test primarily for discrimination based on sexual orientation, indicate that Belgian employers discriminate against young heterosexual women and young heterosexual mothers (Baert 2014). Bygren, Erlandsson, and Gähler (2017) show no difference in employers' hiring preferences for mothers vs. non-mothers and fathers vs. non-fathers, however, which may indicate either that the status value of motherhood varies across countries or that in manifests itself differently across countries.

The status value of any characteristic, including motherhood, varies by culture (Budig, Misra, and Boeckmann 2012). Although we are not aware of any study directly testing the status value of motherhood and fatherhood in Poland, there is evidence available on parents' and non-parents' outcomes on the labor market. Wysieńska-Di Carlo and Karpiński (2015) used POLPAN ${ }^{1}$ data to examine the labor market situation of men and women and describe whether this situation changes with changes in family status (i.e., having children at some point during the occupational career). Their analyses showed that, for both males and females, after controlling for level of education, scores on job complexity and required skills scales, and work experience, the effect of having children on earnings was significant and positive, meaning that having a child was associated with income growth. However, for females, there was a significant and negative interaction between weekly working hours and the number of children. This suggests that, even though an increase in the number of weekly working hours translates into an increase in earnings, this effect is mitigated by the number of children one has - i.e., it is weaker for women with more children. Women working shorter hours actually benefit from having a child, whereas, for women working longer hours, having an additional child has an adverse effect. For males, the interaction effect between weekly working hours and the number of children was not significant. The authors also found that mothers of younger children were at greater risk of losing a job than mothers of older children.

Cukrowska-Torzewska (2016) finds evidence of a motherhood penalty in Poland, especially among mothers with multiple children (the estimates of the penalty are between 8\%-19\% depending on the model specifications; as the results are not always significant, however, they should be treated with caution). On the other hand, Cukrowska-Torzewska and Lovasz (2016) found no motherhood wage penalties, but significant fatherhood premiums - Polish fathers who are full time employees receive wages that are $7.8 \%$ higher than childless men who do not have children.

Recent research regarding attitudes towards women and mothers on the labor market also shows that even though most Polish respondents do not see any negative effects on family life and children of women having a career (they actually tend to view it positively), these same respondents overwhelmingly do not want to work with women and even fewer want a female boss (Centrum Badania Opinii Społecznej 2013).

\section{Motherhood and fatherhood as status characteristics}

Motherhood and fatherhood as status characteristics - theory and predictions

As mentioned above, motherhood is a status characteristic because there is a set of diverse attributes that are evaluated in a particular (devalued) way when linked to a mother than when linked to a non-mother. The notion of a status characteristic is defined in Status Characteristics Theory (Berger and Webster 2006; Ridgeway 2001, 2014). It refers to categorical distinctions that give rise to status beliefs that accord more competence, esteem, and general social worth to one "state" of the underlying characteristic than to other state(s) (Ridgeway 2006). SCT theory distinguishes two types of status characteristics, specific and diffuse, based on the range of situations in which each type becomes relevant. Specific status characteristics are particular abilities that produce well-defined performance expectations relevant to a particular type of task (e.g., years of experience at a given job). A characteristic is diffuse if: (1) the characteristic has two or more states that the actors evaluate differently; (2) there is a set of one or more specific characteristics associated

1. POLPAN is a nationally representative panel survey, which is focused on describing the nation's social structure and changes therein. It has been conducted every five years since 1988 (six waves total, last one in 2013). The original sample in 1988 was 5,817 respondents. Since 1998, new respondents from younger cohorts have been added to insure that the sample remains representative. 
with each state of the diffuse characteristic; and (3) the actor associates a general expectation with each status state. Any characteristic that differentiates between actors, or that is believed to be relevant to the task at hand, will become salient, unless demonstrated to be dissociated from the task (according to the salience and burden of proof assumptions of the theory) (Berger and Webster 2006). Which distinctions carry status value and their specific contents may vary across cultures, but gender, race/ethnicity, age and occupation are almost universally linked to status beliefs.

A specific characteristic is directly relevant to a particular task, whereas a diffuse characteristic is linked to many tasks, although indirectly. Therefore, the weight of each type of characteristic on aggregate performance expectations may differ. When this is the case, status characteristics that are directly relevant to the task may have more weight in forming aggregate expectations than do status characteristics that are not directly relevant.

For motherhood to be a diffuse status characteristic, being a mother needs to represent a level of a distinction that has at least one clearly delineated "comparison" level. Ridgeway and Correll (2004) argue that this level is being a non-mother - i.e. a childless person of any gender or a father who is not primary caregiver (in this view, motherhood is not exclusive to females - a male parent who takes the role of a primary caregiver becomes a "mother").

That would mean, however, that any relevant outcomes (evaluations, rewards, employment decisions, etc.) would have to be compared between "mothers" and everybody else. In contrast, we argue that if a male becomes a primary caregiver for a child, he does not necessarily become a "mother." Expectations towards and evaluations of a male parent are different from those of a female parent regardless of level of involvement in caregiving/parenting (Kmec, Huffman, and Penner 2014). In other words, we argue that both motherhood and fatherhood are status characteristics, that they cannot be simplified to "being a parent" vs. "being childless," and that they are gender specific - i.e., that they interact with expectations regarding gender as a status characteristic.

In most social situations, actors are differentiated by two or more status characteristics, which are often allocated inconsistently — i.e., actors possess a high level of one characteristic, but a low level of another. Sociologists have long been interested in the effects of status inconsistency on judgments, expectations, and behavior. For instance, there is a large literature focused on how status inconsistency affects expectations as to fair or deserved job income (Słomczyński and Wesołowski 2001). According to SCT, in such situations, actors forming their performance expectations combine all the status information that is salient or relevant to the task. According to the principle of organized subsets, all information leading to positive performance expectations is combined by the actor into a subset to determine its positive value, while all information leading to negative performance expectations is combined into a subset to determine its negative value.

As mentioned above, this combining process takes into account that status distinctions differ in terms of their relevance to the immediate task. Status information processing also conforms to the attenuation principle, according to which each additional piece of consistently valued status information will have a smaller marginal effect on one's status advantage or disadvantage. As attenuation occurs within each (positive and negative) subset, the subset with the least elements will be most susceptible to additional information. For instance, a man with a university degree has a status advantage over a woman with the same credential. Yet, suppose that that same man is a surgeon and the woman is a pediatrician. In this case, his status advantage will increase, but this increase will be smaller than that resulting from the activation of gender alone. If the woman, however, is a professor of neonatology and the man is a dental surgeon, his advantage will decrease significantly (Berger et al. 1992).

The aggregated expectations for a given actor are determined by summing the values of the positive and negative subsets. The actor's expectation advantage (or disadvantage) relative to another actor is equal to his or her aggregated expectation minus that of the other actor (Berger and Webster 2006).

Status characteristics not only lead to biased evaluations and divergent expectations (which subsequently guide decisions and behaviors towards persons with particular levels of status distinctions), but they also result in different standards being used to determine whether given performance constitutes proof of ability and competence level. According to Double Standards Theory (Foschi 1996), a stricter standard to prove competence will be applied to a person with lower status, while a more lenient standard will be used for a person with higher status. This is because, when high status individuals perform well, the assessor's expectations are met, and there is no cognitive inconsistency to resolve. When low status individuals perform well, their good performance appears inconsistent with what was anticipated, thus tending to require further 
scrutiny on the part of the assessor (Foschi 2013).

Status characteristics also play a major role in the formation of reward expectations. According to Reward Expectations Theory (Berger et al. 1985), sets of socially validated beliefs (referential structures) are held in common by actors. These beliefs describe what is thought to be the usual association between a valued characteristic and levels of rewards. The theory distinguishes three types of referential structures - categorical, ability, and outcome structures - and describes conditions under which each is activated. The differences between the types of referential structures pertain to the type of information that is linked with reward levels. In the case of categorical referential structures, the information in question is membership in broad social categories, such as gender or motherhood. In the case of ability-based referential structures, the relevant information is a person's ability to perform a task well, or his or her ability to contribute to the group's goal. Finally, outcome-based referential structures link rewards to what one has actually accomplished or achieved (Berger et al. 1985).

These beliefs, when activated, influence the allocation of rewards, which itself is influenced by status situations. There are situations where only performance-relevant skills matter, situations where capacities, accomplishments, and status categories matter, and situations where only status category matters.

A basic corollary of this theory is that if multiple referential beliefs are activated in a situation, actors will combine these structures in forming their reward expectations. Similarly, if multiple status distinctions exist in a situation, actors will combine information from these characteristics.

Based on principles described above, we formulated the following hypotheses:

Hypothesis 1 If higher status is associated with being male rather than female, then a woman working in a high-status occupation represents a case of status inconsistency between gender and occupation. In such cases, all else being equal, the acceptable level of inequality will be higher than in situations of status consistency - i.e., those in which women occupy low and middle positions in the occupational structure. Moreover, both male and female respondents will legitimize such inequality (perceive it as just). This proposition is derived from the joint application of all four theories discussed above.

Hypothesis 2 The above effect will be magnified by the motherhood and fatherhood status. That is, ceteris paribus, being a mother in a high status occupation will be more status inconsistent than being a woman in a high status occupation. Being a father, on the other hand, will have little (but positive) effect on evaluations of just earnings. This hypothesis is consistent with the attenuation principle of SCT, which states that additional information consistent with previous information in a given subset has a declining marginal effect on performance and reward expectations.

Hypothesis 3 Based on the assumption that gender is a salient status characteristic by which men are valued more than women, and assuming that years of job experience constitute a specific status information, we predict that acceptance of inequality of rewards for men and women will be lower for people with more years of labor force experience compared to their less experienced counterparts. This hypothesis is derived from the organized subsets and relevance principles of SCT: Inequalities generated by a diffuse status characteristic can be overcome by the introduction of inconsistent task information. Further, since a task characteristic has greater relevance to the group's goal than a diffuse status characteristic, a task characteristic that is inconsistent with a diffuse status characteristic is predicted to reverse status inequalities. An inconsistent task characteristic might be one that establishes, for example, that a woman possesses greater or equal task competence than a man.

Hypothesis 4 This effect will also be mediated by motherhood and fatherhood status. That is, all else being equal, additional years of experience will have a greater effect in reducing accepted pay differential on mothers than on women with no children. Again, this hypothesis is derived from attenuation and organized subsets principles: for women with no children, information about additional years of experience is consistent with the expectation that childlessness is related to flexibility and being committed to the job. For mothers, in contrast, experience is inconsistent information added to a smaller subset of positive information, and will therefore decrease disadvantage significantly. Additional years of experience will, therefore, have little effect on just reward expectations and evaluations for men.

Hypothesis 5 When men and women work in low-or middle-status occupations, the tolerable level of inequality will be low. This prediction can be derived from DST and shifting standard theory. According to $D S T$, there is no status inconsistency, so the evidence of ability will not be subject to an assessor's scrutiny. 
Hypothesis 6 The relationship specified in Hypothesis 5, ceteris paribus, will also vary by motherhood and fatherhood status, although the magnitude of the difference will be rather small in absolute terms. Having children will have a positive effect on perceived just incomes for fathers and a slight negative effect for mothers. (Consistent with the principles described above, additional information about having children will be inconsistent information added to a smaller subset of positively valued status elements in case of men, and, in the case of women, it will be an additional piece of consistent information added to a larger subset of negatively valued elements.)

\section{Methods}

In order to test our hypotheses, we used a vignette (multifactorial) design. An important advantage of using vignettes in sociological research is that they allow for obtaining judgments from respondents concerning combinations of characteristics that occur rather rarely in the population (Heise 2010, chap. 4; Wallander 2009). Also, to study status inconsistency effects on the perceptions of justice of earnings, one needs a reasonably large number of status-inconsistent situations in order to be able to compare them systematically with status-consistent ones. Thus, the result of the test depends, among other things, on the relative frequency of status inconsistency in the population under study. In a vignette study, this is of little concern because the combination of characteristics assigned to a vignette is subject to explicit manipulation.

In the present study, we followed the approach of Jasso and Webster (1999). Unlike earlier vignette studies on justice of earnings, in which a single amount of earnings was assigned to each vignette, Jasso and Webster assigned multiple amounts to a single vignette and participants were asked to evaluate the justice of each amount given the characteristics of the vignette. The framework is based on Jasso's justice evaluation function and allows for estimation of just earnings for each vignette (Jasso 2007). Jasso recommends using this estimation procedure, which she also calls the "indirect method," as opposed to asking subjects directly how much they think persons described in the vignettes should earn, as these responses are likely to be affected by individual expressiveness and other confounding factors (Whitmeyer 2004). That is, instead of asking about just earnings directly, Jasso and Webster (1999) propose to ask the subjects to assess a series of hypothetical amounts reflecting how fair or unfair each of these rewards would be for a particular person. As Jasso and Webster (1999) explain in detail, by studying the magnitudes of injustice felt by the respondents as a result of the assessment, we can learn how far each of the amounts departs from the "true just amount" - and in which direction. This way, we can retrieve the "true just amount" from the evaluations (see Jasso 2007; Jasso and Webster 1999, for more details).

\subsection{Vignettes' sample}

With 2 gender categories, 3 parenthood statuses, 8 ages, and 21 occupational titles $^{2}$, there are $2 \times 3 \times 8 \times 21=$ 1,008 possible combinations. Removing combinations that are logically improbable reduced the total number of available vignettes to $996 .{ }^{3}$ From that a random sample of 21 vignettes was assigned to each respondent using a stratified sampling, with each occupation being a stratum. As explained above, our interest was in the effects of status inconsistency on the perceptions of just earnings. Thus, we classified vignettes in our population as consistent and inconsistent, depending on the combination of the categories of gender and occupational prestige.

To summarize, respondents were presented with fictitious descriptions of a full-time employee of certain gender, age, parenthood status, education, job experience, and occupation. A random sample of vignettes was shown to respondents (21 to each study participant), each of whom was asked to evaluate them in terms of the "appropriateness" of six earnings randomly assigned to the vignettes.

2. Occupations were differentiated into high-, medium-, and low-prestige categories. The basis of the differentiation is a recent scale of occupational prestige proposed by (Domański, Słomczyński, and Sawiński 2009). Seven occupations belonging to each status category were used.

3. One of the occupations that we used in our vignettes was the professor of early education. Because it is unlikely to obtain professorship by the age of 40 in the Polish academic system, we decided to remove all the vignettes in which the job a professor was combined with an age younger than 40 . 
Figure 1: Example of a vignette

A 35 year old woman, mother of two. She works as a nurse and her overall job experience is 7 years.

To what extent do you think the incomes below would be appropriate for this person?

For each income please indicate whether you think it is appropriate, too low, too high.

Please place each amount in a box that corresponds to your opinion.

Depending on your feelings, you can place each amount in different box or several amounts in the same box leaving some boxes empty.

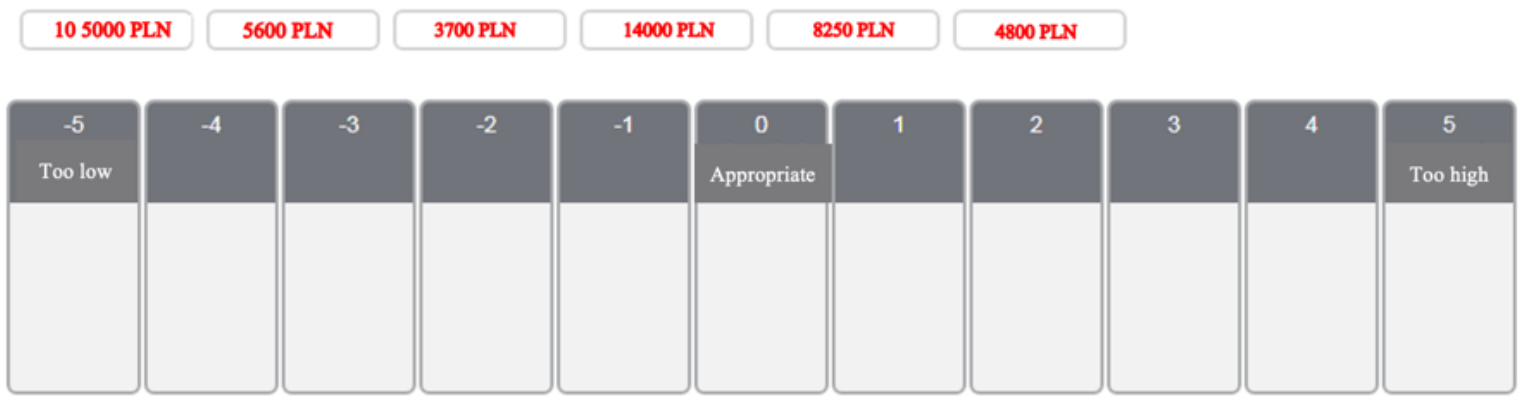

\subsection{Respondent sample}

The vignette survey was administered to a sample of registered members of an Internet panel run by one of the leading marketing and opinion research companies $(\mathrm{n}=2001$ respondents $)$ in Poland in 2017. The sample was not random, but it was designed as to match the composition of Polish working population along age, gender, education, and geographical location. Approximately half of our respondents were female, with a mean age of 41.6 years.

\subsection{Analytical Strategy}

Obtaining the estimates of just earnings is only the first step toward our main goal of testing the hypotheses developed in the previous section. Once obtained, the estimates are used as a dependent variable in a series of subsequent multilevel regression models that will allow us to conduct those tests formally. Our initial model is defined as follows:

$$
\begin{aligned}
y_{i j} & =\beta_{0 j}+\beta_{1} f_{i}+\beta_{2} a_{i}+\beta_{3} a_{i}^{2}+\beta_{4} l_{i}+\beta_{5} h_{i}+\beta_{6} c_{1 i}+\beta_{7} c_{2 i}+\epsilon_{i j} \\
\beta_{0 j} & =\gamma_{00}+\sum_{k=1}^{K} \gamma_{0 k} z_{j} k+r_{0 j}
\end{aligned}
$$

where $y_{i j}$ stands for the (logged) amount of earnings that respondent $j$ believes are a fair pay for rewardee $i$. The quantities $f_{i}, a_{i}, a_{i}^{2}, l_{i}, u_{i}, c_{1 h}, c_{2 h}$ correspond to rewardee $i$ 's characteristics, that is, gender, age, age squared, the dummy for working in low status occupations, the dummy for working in high status occupations, the dummy for having 1 child, and the dummy for having 2 or more children, respectively. The multilevel character of our data is represented by the intercept in (1a), which, as indicated by the double subscript, is allowed to vary across respondents and is modeled as dependent upon respondent-level characteristics, which are denoted by variables $z_{1}$ through $z_{K}$ in the second equation in (1b). These variables are not listed here, but they include, among others, gender, age, education, earnings, work situation, marital status, and the number of children. 
While the initial model (1) provides a useful starting point for our analysis, it is not sufficient for testing our hypotheses. This requires models with complex two-way and three-way interaction effects. That is, in order to test our hypotheses, we have to extend the initial model by adding interactions between variables representing gender and occupational prestige. This results in the following extension of (1):

$$
\begin{aligned}
y_{i j} & =\beta_{0 j}+\beta_{1} f_{i}+\beta_{2} a_{i}+\beta_{3} a_{i}^{2}+\beta_{4} l_{i}+\beta_{5} h_{i}+\beta_{6} c_{1 i}+\beta_{7} c_{2 i}+\beta_{8} f_{i} \times l_{i} \\
& +\beta_{9} f_{i} \times u_{i}+\epsilon_{i j} \\
\beta_{0 j} & =\gamma_{00}+\sum_{k=1}^{K} \gamma_{0 k} z_{j k}+r_{0 j}
\end{aligned}
$$

The only difference between (1) and (2) is the inclusion of the interaction terms $f_{h} \times l_{h}$ and $f_{h} \times u_{h}$ in the former. Because of this, the predicted magnitude of the just gender pay gap will be different at different levels of occupational structure. To see this, consider two rewardees, $h$ and $i$ who work in high-prestige occupations, have the same number of children, and are of the same age, but $h$ is female while $i$ is male. Thus, we have $f_{h}=1, f_{i}=0, u_{h}=1, h_{i}=1, l_{h}=0, l_{i}=0$ which, after substituting the quantities in (2) leads to:

$$
\begin{aligned}
y_{h j} & =\beta_{0 j}+\beta_{1}+\beta_{2} a+\beta_{3} a^{2}+\beta_{5}+\beta_{6} c_{1}+\beta_{7} c_{2}+\beta_{9} \\
y_{i j} & =\beta_{0 j}+\beta_{2} a+\beta_{3} a^{2}+\beta_{5}+\beta_{6} c_{1}+\beta_{7} c_{2}
\end{aligned}
$$

Subtracting the latter equation from the former, we get $\beta_{1}+\beta_{9}$ as our estimate of the just gender pay gap in high-prestige occupations. This is to say that, according to these formulas, just earnings for women are $\exp \left(\beta_{1}+\beta_{9}\right)$ just earnings for men (because we use the logged amounts of just earnings in the analysis). Also, $\beta_{1}+\beta_{9}<0$ implies that women working in high-prestige occupations are entitled to lower earnings than otherwise comparable men (in the eyes of our respondents). In a similar way, the estimates of the gap at medium-prestige and low-prestige occupations are predicted to be $\beta_{1}$ and $\beta_{1}+\beta_{8}$, respectively.

However, our first hypothesis claims not only that women are generally viewed as less deserving than men, but also that the magnitude of the just gender pay gap is greater in high-prestige occupations than in medium or low-prestige occupations. That is, the hypothesis predicts that

$$
\begin{aligned}
& \beta_{1}+\beta_{9}<0, \beta_{1}<0 \text { and } \beta_{1}+\beta_{8}<0, \\
& \beta_{1}+\beta_{9}<\beta_{1}, \text { which implies that } \beta_{9}<0, \\
& \beta_{1}+\beta_{9}<\beta_{1}+\beta_{8}, \text { which implies that } \beta_{9}<\beta_{8}
\end{aligned}
$$

Table 1: Parameters estimates for the multi-level models with the log of just earnings as the dependent variable and interactions between prestige, gender, and parenthood status

\begin{tabular}{lccc}
\hline & Model 1 & Model 2 & Model 3 \\
\hline Female & $-0.015^{*}$ & -0.003 & 0.002 \\
& $(0.007)$ & $(0.012)$ & $(0.020)$ \\
Age & $0.017^{* * *}$ & $0.017^{* * *}$ & $0.017^{* * *}$ \\
& $(0.003)$ & $(0.003)$ & $(0.003)$ \\
Age $^{2}$ & $-0.000^{* * *}$ & $-0.000^{* * *}$ & $-0.000^{* * *}$ \\
& $(0.000)$ & $(0.000)$ & $(0.000)$ \\
Prestige: low & $-0.162^{* * *}$ & $-0.154^{* * *}$ & $-0.167^{* * *}$ \\
& $(0.008)$ & $(0.012)$ & $(0.020)$ \\
Prestige: high & $0.186^{* * *}$ & $0.195^{* * *}$ & $0.217^{* * *}$ \\
& $(0.008)$ & $(0.012)$ & $(0.021)$ \\
1 child & -0.001 & -0.001 & 0.002 \\
& $(0.008)$ & $(0.008)$ & $(0.020)$ \\
2 or more children & $0.024^{* *}$ & $0.024^{* *}$ & 0.035 \\
& $(0.008)$ & $(0.008)$ & $(0.020)$ \\
\hline
\end{tabular}




\begin{tabular}{|c|c|c|c|}
\hline & Model 1 & Model 2 & Model 3 \\
\hline Female $\times$ Prestige: low & & $\begin{array}{r}-0.016 \\
(0.017)\end{array}$ & $\begin{array}{r}-0.000 \\
(0.029)\end{array}$ \\
\hline Female $\times$ Prestige: high & & $\begin{array}{r}-0.019 \\
(0.017)\end{array}$ & $\begin{array}{r}-0.040 \\
(0.029)\end{array}$ \\
\hline Female $\times 1$ child & & & $\begin{array}{r}-0.003 \\
(0.029)\end{array}$ \\
\hline Female $\times 2$ or more children & & & $\begin{array}{c}-0.012 \\
(0.029)\end{array}$ \\
\hline Prestige: low $\times 1$ child & & & $\begin{array}{c}0.019 \\
(0.029)\end{array}$ \\
\hline Prestige: high $\times 1$ child & & & $\begin{array}{c}-0.019 \\
(0.029)\end{array}$ \\
\hline Prestige: low $\times 2$ or more children & & & $\begin{array}{c}0.018 \\
(0.029)\end{array}$ \\
\hline Prestige: high $\times 2$ or more children & & & $\begin{array}{r}-0.045 \\
(0.029)\end{array}$ \\
\hline Female $\times$ Prestige: low $\times 1$ child & & & $\begin{array}{c}-0.023 \\
(0.041)\end{array}$ \\
\hline Female $\times$ Prestige: high $\times 1$ child & & & $\begin{array}{c}0.014 \\
(0.041)\end{array}$ \\
\hline Female $\times$ Prestige: low $\times 2$ or more children & & & $\begin{array}{c}-0.024 \\
(0.041)\end{array}$ \\
\hline Female $\times$ Prestige: high $\times 2$ or more children & & & $\begin{array}{c}0.049 \\
(0.041) \\
\end{array}$ \\
\hline $\mathrm{AIC}$ & -110715.835 & -110700.381 & -110628.665 \\
\hline $\mathrm{BIC}$ & -110621.783 & -110589.229 & -110432.012 \\
\hline Log Likelihood & 55368.917 & 55363.191 & 55337.333 \\
\hline Num. obs. & 38183 & 38183 & 38183 \\
\hline Num. groups: respid & 1964 & 1964 & 1964 \\
\hline Num. groups: vignid & 996 & 996 & 996 \\
\hline Var: respid (Intercept) & 0.095 & 0.095 & 0.095 \\
\hline Var: vignid (Intercept) & 0.012 & 0.012 & 0.012 \\
\hline Var: Residual & 0.002 & 0.002 & 0.002 \\
\hline
\end{tabular}

${ }^{* * *} p<0.001 ;{ }^{* *} p<0.01 ;{ }^{*} p<0.05$

Table 1 shows parameter estimates for three models: the initial model (1) (see column 1 in Table 1); the current model (2) that we use to test Hypothesis 1 (see column 2); and a more complex model (see column 3 ), which we discuss in the next section. Because the total number of parameters in the models is rather large, the table shows only the estimates of the parameters that are relevant for testing our hypotheses.

The estimate of the main effect of rewardee's gender is -0.0032 in the current model. This effect is weak in absolute terms and not statistically significant at the 0.05 level; we don't have sufficient evidence to conclude that women working in medium-prestige occupations are seen by our survey participants as less deserving of pay than otherwise identical men.

Let us now consider rewardees in high-prestige occupations. As demonstrated earlier, the just gender pay gap at the high level of occupational prestige is $\beta_{1}+\beta_{9}$, which, using the estimates in Table 1 , is equal to $-0.0032-0.0192=-0.0224$. The interpretation of the estimate is that our respondents view women working in high-prestige occupations as deserving $\exp (-0.0224)=0.978$ of the just pay of male rewardees working in high-prestige occupations. This result, while not statistically significant at the conventional level (test statistic: 3.5 , with a chi-square distribution and based on 1 degree of freedom and $p=0.06$ ), is nevertheless 
consistent with the first hypothesis. That is, our respondents perceive women working in high-prestige occupation as deserving slightly less than otherwise comparable men.

As for the low-prestige occupations, the just gender pay gap is equal to $\beta_{1}+\beta_{8}$, as explained earlier. Using the estimates in Table 1 we find it is equal to $-0.0032-0.0161=-0.0193$. This means that participants in our survey believe that women working in low-prestige occupations deserve to be paid $2 \%$ less than otherwise identical men. This is not large in magnitude and the pay gap reaches significance at the level of $p=0.1$ only. Finally, we don't have enough evidence to conclude that the magnitude of the just gender pay gap is greater at the high level of occupational structure than at the low level: testing the hypothesis that $\beta_{9}<\beta_{8}$ yields a chi-square test statistic of 0.03 with $\mathrm{df}=1$, resulting in $p=0.86$.

Our second hypothesis links differences in perceptions of just pay for men and women with their parenthood status. In other words, because being a mother adds another (i.e., in addition to being a female) piece of information that is inconsistent with working in a high-prestige occupation, our second hypothesis predicts that mothers will be seen by our subjects as deserving lower pay than otherwise identical women who don't have children. For men, being a father is not inconsistent with doing a high-prestige job, so it will have only a moderate effect on expectations of just pay for men, according to the attenuation principle of status characteristics theory. As a result, the just gender pay gap for rewardees with children is hypothesised to be higher than for childless rewardees.

That is, not only will the effect of gender vary by occupational status, but the effect of parenthood will also vary depending on gender and occupational status. In order to test this hypothesis we have to extend the previous model by adding two-way interaction terms between parenthood and occupational status and three-way interaction terms between gender, parenthood, and occupational status:

$$
\begin{aligned}
y_{i j} & =\beta_{0 j}+\beta_{1} f_{i}+\beta_{2} a_{i}+\beta_{3} a_{i}^{2}+\beta_{4} l_{i}+\beta_{5} u_{i}+\beta_{6} c_{1 i}+\beta_{7} c_{2 i}+\beta_{8} f_{i} \times l_{i} \\
& +\beta_{9} f_{i} \times u_{i}+\beta_{10} f_{i} \times c_{1 i}+\beta_{11} f_{i} \times c_{2 i}+\beta_{12} c_{1 i} \times l_{i}+\beta_{13} c_{1 i} \times u_{i} \\
& +\beta_{14} c_{2 i} \times l_{i}+\beta_{15} c_{2 i} \times u_{i}+\beta_{16} f_{i} \times c_{1 i} \times l_{i}+\beta_{17} f_{i} \times c_{1 i} \times u_{i} \\
& +\beta_{18} f_{i} \times c_{2 i} \times l_{i}+\beta_{19} f_{i} \times c_{2 i} \times u_{i}+\epsilon_{i} j \\
& \beta_{0 j}=\gamma_{00}+\sum_{k=1}^{K} \gamma_{0 k} z_{j k}+r_{0} j
\end{aligned}
$$

Now, this model may look somewhat complex, but the only difference between it and the previous one are the interaction terms with coefficients $\beta_{10}$ through $\beta_{19}$. Adding these terms, of course, affects the calculation of just gender pay gaps. For instance, consider again rewardess $h$ and $i$ who are of the same age and have the same job experience, and both also work in high-prestige occupations and has just one child. The only difference is that $h$ is a woman and $i$ is a man. Thus, variables $U$ and $C_{1}$ are set to 1 for both rewardees, variables $L$ and $C_{2}$ are set to 0 , and $f_{h}=1$, while $f_{i}=0$. After appropriate substitutions, we arrive at the following formulas for predicted logs of just earnings for $h$ and $i$, respectively:

$$
\begin{aligned}
y_{h j} & =\beta_{0 j}+\beta_{1}+\beta_{2} a+\beta_{3} a^{2}+\beta_{5}+\beta_{6}+\beta_{9}+\beta_{10}+\beta_{13}+\beta_{17} \\
y_{i j} & =\beta_{0 j}+\beta_{2} a+\beta_{3} a^{2}+\beta_{5}+\beta_{6}+\beta_{13}
\end{aligned}
$$

Subtracting the latter formula from the former yields $\beta_{1}+\beta_{9}+\beta_{10}+\beta_{17}$ as the estimate of the (logged) just gender pay gap for rewardees with exactly 1 child who work in a high-prestige occupation. By the same token, the estimate of just gender pay gap for rewardees with 2 or more children is equal to $\exp \left(\beta_{1}+\beta_{9}+\right.$ $\left.\beta_{11}+\beta_{19}\right)$. Thus, the first child multiplies the just gender pay gap for rewardees in high-prestige occupations by $\exp \left(\beta_{10}+\beta_{17}\right)$, and having 2 or more children - by $\exp \left(\beta_{11}+\beta_{19}\right)$.

In a similar manner, the just gender pay gap for rewardees in medium-prestige occupations can be shown to be just $\exp \left(\beta_{1}+\beta_{10}\right)$ for rewardees with just 1 child and $\exp \left(\beta_{1}+\beta_{11}\right)$ for those with 2 or more children. Finally, for employees in the low-prestige occupations, the just gender pay gaps are estimated as $\exp \left(\beta_{1}+\beta_{8}+\beta_{10}+\beta_{16}\right)$ for 1 child and $\exp \left(\beta_{1}+\beta_{8}+\beta_{11}+\beta_{18}\right)$ for 2 or more children. The results of fitting the present model to our data are shown in Table 1 , column (3).

To make the interpretation of the results easier, we also plotted predicted gender pay gaps for each category of parental status and occupational prestige (see Figure 2). These gaps are expressed in terms of the ratio of female to male just earnings, so that the value of 1 corresponds to just female earnings 
Figure 2: Just gender pay ratio by occupational prestige and the number of children
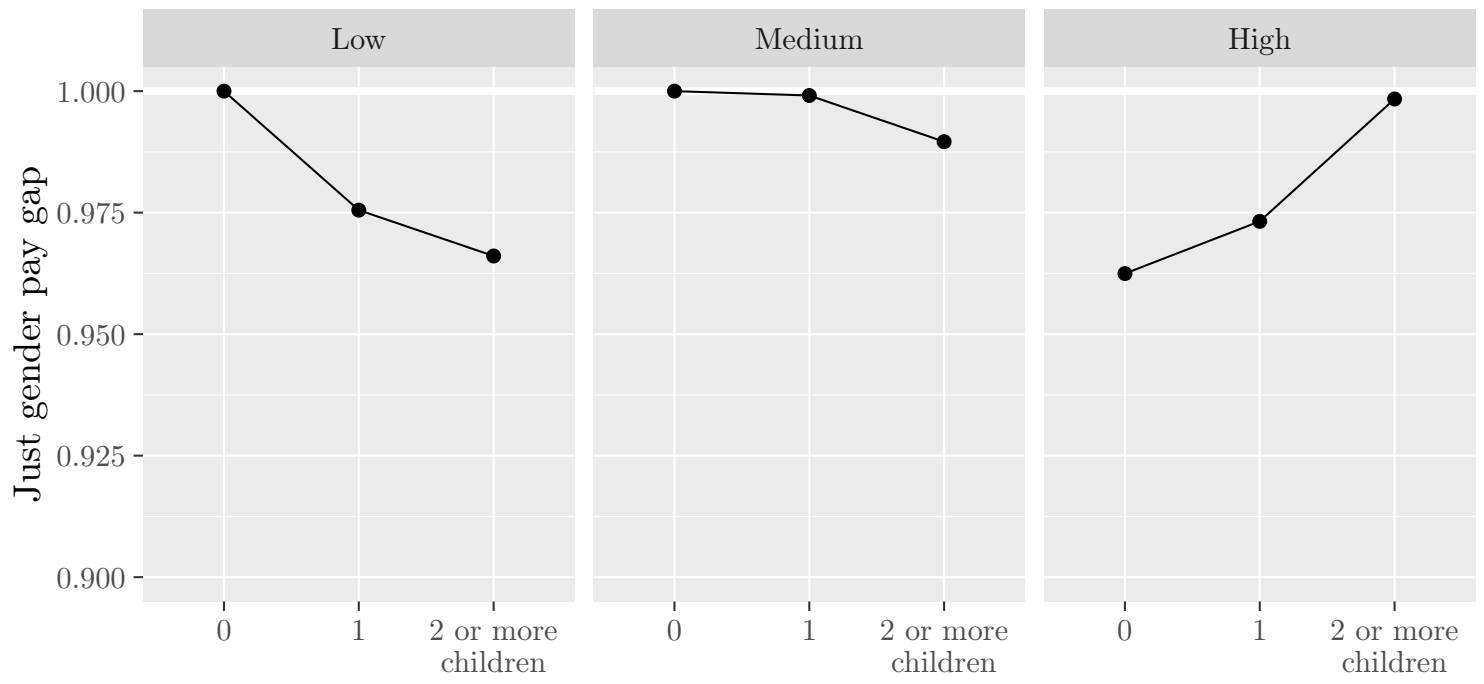

The number of children

matching exactly those of otherwise identical men; values greater than 1 correspond to just earnings for women exceeding those for men; and values below 1 correspond to just earnings for women being smaller than for men. As we can see, the effect of parenthood status varies by the category of occupational prestige: for rewardees in the low-prestige jobs, the width of the gap increases with the number of children, but it decreases with the number of children in high-prestige occupations. The gap is virtually non-existent for childless employees in the low-prestige occupations, but it drops to 0.965 for those with 2 or more children, meaning that women working in low-prestige jobs who have 2 or more children are seen as entitled to pay that is $96.5 \%$ of that considered just for otherwise identical men.

As regards high-prestige occupations, the just pay gap for childless employees is estimated to be equal to 0.962 . However, the width of the gap shrinks with the number of children. It is 0.973 for high-prestige rewardees with 1 child and to 0.998 for those with 2 or more children.

In an additional analysis, we also estimated a multi-level model with interaction effects involving age (both linear and quadratic components). This way, we were able to see how age modifies the effects of gender and parenthood on the predicted inequality of pay between men and women. Because the models include three-way interactions between age, gender, and parenthood, we summarise the results of the model graphically (see Figure 3).

The predicted just gender pay gap varies with age, but the relationship is different depending on parenthood status. Let us first look at the line representing rewardees with no children. For the youngest and the oldest rewardees in that category, the pay gap is actually in favor of women, even though the size of the advantage is very small. But, between the ages of 33 and 62 , the pay gap is to women's disadvantage, reaching the minimum at around the age of 47 . For rewardees with exactly one child, the just gender pay gap follows a similar pattern, although, it is consistently below 1 . When they are around 50 , female rewardees with 1 child are seen by our respondents as deserving earnings $2.5 \%$ lower than otherwise identical men. The pattern for respondents with 2 or more children is strikingly different. Unlike in the two other categories, the gap is initially (i.e., for rewardees at the age of 30) relatively wide (about 3.5\%). Beyond that, it begins to shrink, but past the age of 55 it starts to widen again. In general, these results give some support to our predictions, showing that acquiring job experience can reduce the inequality, but only applied to rewardees with two or more children. 
Figure 3: Just gender pay ratio by age and the number of children
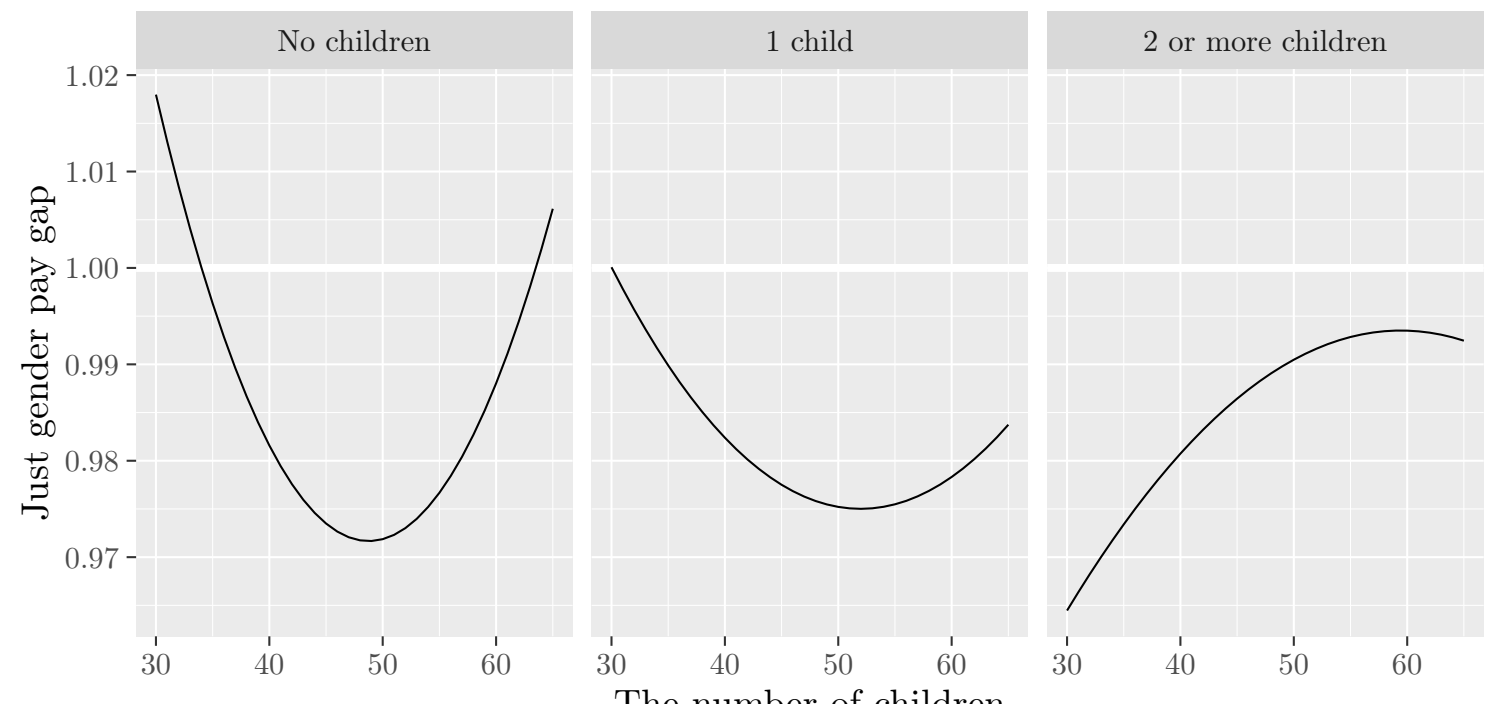

\section{Conclusion and discussions}

The results of our experiment only partially conformed to our predictions. That is, we observed gender effects on perceptions of just earnings gaps, which varied by occupational status. Parenthood status, on the other hand, varied only by age. One of the reasons might be that, while we controlled for occupational prestige, we did not examine the effects of varying years of experience (which is a directly relevant status characteristic). We also do not analyze here effects of gender composition and typification of occupations.

That said, our results indicate that individuals' perceptions of just earnings in Poland are influenced by the gender and parenthood status of the earners being evaluated, but occupational status might not be as important as we had anticipated. These results are nonetheless largely consistent with status characteristics theory, and this is the first evidence of this in Poland.

A follow-up experiment in which we varied experience and introduced measures to test for the status effects in female/male dominated occupations and gendered-neutral vs. gender-typed occupations is underway.

\section{References}

Abendroth, Anja-Kristin, Matt L. Huffman, and Judith Treas. 2014. "The parity penalty in life course perspective: Motherhood and occupational status in 13 European countries." American Sociological Review 79 (5): 993-1014.

Aisenbrey, Silke, Marie Evertsson, and Daniela Grunow. 2009. "Is There a Career Penalty for Mothers' Time Out? A Comparison of Germany, Sweden and the United States." Social Forces 88, no. 2 (December): 573-605. ISSN: 0037-7732. doi:10.1353/sof.0.0252. eprint: https://academic .oup. com/sf/articlepdf/88/2/573/6884763/88-2-573.pdf. https://doi.org/10.1353/sof.0.0252.

Auspurg, Katrin, Thomas Hinz, and Carsten Sauer. 2017. "Why should women get less? Evidence on the gender pay gap from multifactorial survey experiments." American Sociological Review 82 (1): 179-210.

Baert, Stijn. 2014. "Career lesbians. Getting hired for not having kids?" Industrial Relations Journal 45 (6): 543-561. doi:10.1111/irj.12078. eprint: https://onlinelibrary.wiley.com/doi/pdf/10.1111/ irj.12078. https://onlinelibrary.wiley.com/doi/abs/10.1111/irj.12078. 
Berger, Joseph, M. Hamit Fisek, Robert Z. Norman, and David G. Wagner. 1985. "Formation of Reward Expectations in Status Situations." In Status, Rewards, and Influence, edited by Joseph Berger and Morris Zelditch, Jr., 215-261. San Francisco: Jossey-Bass.

Berger, Joseph, Robert Z. Norman, James W. Balkwell, and Roy F. Smith. 1992. "Status inconsistency in task situations: A test of four status processing principles." American Sociological Review 57:843-855.

Berger, Joseph, and Murray Webster Jr. 2006. "Expectations, Status, and Behavior." In Contemporary Social Psychological Theories, edited by Peter J. Burke. Stanford: Stanford University Press.

Budig, Michelle J., and Paula England. 2001. "The Wage Penalty for Motherhood." American Sociological Review 66:204-225.

Budig, Michelle J., and Melissa J. Hodges. 2010. "Differences in Disadvantage: Variation in the Motherhood Penalty across White Women's Earnings Distribution." American Sociological Review 75 (5): 705-728. doi:10 . 1177/0003122410381593. eprint: https : / / doi .org/10 .1177/0003122410381593. https : //doi.org/10.1177/0003122410381593.

Budig, Michelle J., Joya Misra, and Irene Boeckmann. 2012. "The motherhood penalty in cross-national perspective: The importance of work-family policies and cultural attitudes." Social Politics 19 (2): 163193.

. 2016. "Work-family policy trade-offs for mothers? Unpacking the cross-national variation in motherhood earnings penalties." Work and Occupations 43 (2): 119-177.

Bygren, Magnus, Anni Erlandsson, and Michael Gähler. 2017. "Do Employers Prefer Fathers? Evidence from a Field Experiment Testing the Gender by Parenthood Interaction Effect on Callbacks to Job Applications." European Sociological Review 33, no. 3 (May): 337-348. ISSN: 0266-7215. doi:10.1093/ esr/jcx051. eprint: https://academic.oup.com/esr/article-pdf/33/3/337/17691942/jcx051. pdf. https://doi.org/10.1093/esr/jcx051.

Centrum Badania Opinii Społecznej. 2013. Kobieta pracujaca (Working women). Warszawa. https://www . cbos.pl/SPISKOM.POL/2013/K_028_13.PDF.

Cooke, Lynn Prince. 2014. "Gendered parenthood penalties and premiums across the earnings distribution in Australia, the United Kingdom, and the United States." European Sociological Review 30 (3): 360-372.

Correll, Shelley J, Stephen Benard, and In Paik. 2007. "Getting a job: Is there a motherhood penalty?" American Journal of Sociology 112 (5): 1297-1338.

Cukrowska-Torzewska, Ewa. 2016. "How much does it cost to rear children in Poland?" European Journal of Women's Studies 23 (2): 200-216.

Cukrowska-Torzewska, Ewa, and Anna Lovasz. 2016. "Are children driving the gender wage gap? Comparative evidence from Poland and Hungary." Economics of Transition 24 (2): 259-297.

Domański, Henryk, Kazimierz M. Słomczyński, and Zbigniew Sawiński. 2009. Sociological Tools Measuring Occupations: New Classification and Scales. Warsaw: IFiS Publishers.

England, Paula, Jonathan Bearak, Michelle J. Budig, and Melissa J. Hodges. 2016. "Do highly paid, highly skilled women experience the largest motherhood penalty?" American Sociological Review 81 (6): 11611189.

Evertsson, Marie, and Daniela Grunow. 2012. "Women's work interruptions and career prospects in Germany and Sweden." International Journal of Sociology and Social Policy 32 (9/10): 561-575.

Foschi, Martha. 1996. "Double Standards in the Evaluation of Men and Women." Social Psychology Quarterly 59:237-254.

_. 2013. "Double standards for competence (In Polish)." In Group processes in a sociological perspective, edited by Kinga Wysieńska and Joanna Heidtman, 225-239. Warsaw: Scholar. 
Gangl, Markus, and Andrea Ziefle. 2009. "Motherhood, labor force behavior, and women's careers: An empirical assessment of the wage penalty for motherhood in britain, germany, and the united states." Demography 46 (2): 341-369.

Glauber, Rebecca. 2008. "Race and Gender in Families and at Work: The Fatherhood Wage Premium." Gender 8 Society 22 (1): 8-30.

Heise, David R. 2010. Surveying Cultures: Discovering Shared Conceptions and Sentiments. New York: John Wiley \& Sons.

Hodges, Melissa J., and Michelle J. Budig. 2010. "Who Gets the Daddy Bonus?: Organizational Hegemonic Masculinity and the Impact of Fatherhood on Earnings." Gender \&3 Society 24 (6): 717-745.

Jasso, Guillermina. 2007. "Studying Justice: Measurement, Estimation, and Analysis of the Actual Reward and the Just Reward." In Distributive and Procedural Justice: Research and Social Applications, edited by Kjell Törnblom and Riel Vermunt. London: Ahsgate.

Jasso, Guillermina, and Murray Webster Jr. 1997. "Double standards in just earnings for male and female workers." Social Psychology Quarterly 60 (1): 66-78.

_ 1999. "Assessing the gender gap in just earnings and its underlying mechanisms." Social Psychology Quarterly 62 (4): 367-380.

Kmec, Julie A., Matt L. Huffman, and Andrew M. Penner. 2014. "Being a Parent or Having a Parent? The Perceived Employability of Men and Women Who Take Employment Leave." American Behavioral Scientist 58 (3): 453-472. doi:10 . 1177 / 0002764213503338. eprint: https : / / doi .org/10 . 1177 / 0002764213503338 . https://doi.org/10.1177/0002764213503338.

Koslowski, Alison Smith. 2010. "Working Fathers in Europe: Earning and Caring." European Sociological Review 27, no. 2 (February): 230-245. ISSN: 0266-7215. doi:10.1093/esr/ jcq004. eprint: https : //academic . oup.com/esr/article-pdf/27/2/230/1178018/jcq004.pdf. https://doi.org/10. 1093/esr/jcq004.

Magnusson, Charlotta. 2010. "Why Is There a Gender Wage Gap According to Occupational Prestige?: An Analysis of the Gender Wage Gap by Occupational Prestige and Family Obligations in Sweden." Acta Sociologica 53 (2): 99-117. doi:10 .1177/0001699310365627. eprint: https : // doi .org/10 .1177/ 0001699310365627 . https://doi.org/10.1177/0001699310365627.

Petersen, Trond, Andrew M. Penner, and Geir Høgsnes. 2014. "From Motherhood Penalties to Husband Premia: The New Challenge for Gender Equality and Family Policy, Lessons from Norway." American Journal of Sociology 119 (5): 1434-1472. doi:10.1086/674571. eprint: https ://doi .org/10.1086/ 674571. https://doi.org/10.1086/674571.

Ridgeway, Cecilia L, and Shelley J. Correll. 2004. "Motherhood as a status characteristic." Journal of Social issues 60 (4): 683-700.

Ridgeway, Cecilia L. 2001. "Inequality, Status, and the Construction of Status Beliefs." In Handbook of Sociological Theory, edited by Jonathan Turner, 323-340. New York: Springer.

. 2006. "Status Construction Theory." In Contemporary Social Psychological Theories, edited by Peter J. Burke, 301-323. Stanford: Stanford University Press.

2011. Framed by Gender: How Gender Inequality Persists in the Modern World. New York: Oxford University Press.

2014. "Why Status Matters for Inequality?" American Sociological Review 79:1-16.

Słomczyński, Kazimierz M., and Włodzimierz Wesołowski. 2001. "Distributive Justice and Status (In)consistency: A Theoretical Debate and Empirical Evidence." Polish Sociological Review 135:299-312. 
Susan, Harkness, and Waldfogel Jane. 2003. "The family gap in pay: Evidence from seven industrialized countries." In Worker Well-Being and Public Policy, edited by Solomon W. Polachek, 22:369-413. Emerald Group Publishing Limited. ISBN: 978-1-84950-213-9, 978-0-76231-026-5/0147-9121.

Wallander, Lisa. 2009. "25 Years of Factorial Surveys in Sociology: A Review." Social Science Research 38 (3): 505-520. ISSN: 0049-089X. doi:https : // doi .org/10.1016/j. ssresearch . 2009.03.004. http://www.sciencedirect.com/science/article/pii/S0049089X09000192.

Whitmeyer, Joseph M. 2004. "Past and Future Applications of Jasso's Justice Theory." Sociological Theory 22 (3): 432-444.

Wysieńska-Di Carlo, Kinga, and Zbigniew Karpiński. 2015. "Is The Motherhood Penalty Real? The Evidence From Poland." Accessed October 24, 2019. https://www. shankerinstitute.org/blog/motherhoodpenalty-real-evidence-poland. 